\title{
ZigBee Investigation Within Smart Micro Grid
}

\author{
Elkhorchani Habib ${ }^{1}$, Grayaa Khaled ${ }^{2}$ \\ Phd, Université de Tunis, ENSIT, CEREP, Tunis, 1001, Tunisia ${ }^{1}$ \\ Pr, Université de Tunis, ENSIT, SYSCOM/ENIT, Tunis, 1001, Tunisia ${ }^{2}$
}

\begin{abstract}
Nowadays, the current energy grid is evolving into a smart grid. Communications research, especially wireless communications research, is considered as part of a bigger challenge and is seen as a great enabler for future Smart Grids. Wireless Sensor Network (WSN) has drawn wide attention on communicating data for Smart Grid and domestic energy management systems. In order to address this issue, this paper presents some of the challenges of WSN system based on the ZigBee technology (IEEE 802.15.4) in Smart Micro Grid. The simulation results with Opnet Modeler obtained show that the application of the WSN based on the ZigBee technology may be very promising in several Smart Micro Grids and Smart Grids applications such as the energy control and management. In this work, we also investigate the experimental performance of ZigBee based on measurements of the Received Signal Strength Indicator (RSSI) in different parts of the Smart Home. Such models help modelling and sizing Micro Grid systems.
\end{abstract}

Keywords: Smart Micro Grid; Smart Home; WSN; ZigBee, RSSI.

\section{INTRODUCTION}

The integration of smart devices and an intelligent The access mode for all these frequency bands is Direct network in a home can make it smart. To better control Sequence Spread Spectrum (DSSS). The $2450 \mathrm{MHz}$ band energy consumption in the Smart Home, the Home Area employs Offset Quadrature Phase Shift Keying (O-QPSK) Network (HAN) is used to collect sensor information from for modulation while the 868/915 MHz bands use Binary a variety of devices inside the home, and optionally sends Phase Shift Keying (BPSK). Table I summarizes the control information to it [1]. The structure of Wireless frequency bands and data rates of the ZigBee technology Sensor Networks (WSN) in the smart home requires three IEEE 802.15.4 [8]. different devices: Coordinator, Router and End Device. All home environments can then be monitored and the required data can be delivered to the coordinator. After the treatment of the data, the appropriate control commands are sent through coordinator to home appliances or the renewable energy sources that surround the home $[1,2]$.

In this study, we use ZigBee protocol since it is widely used for WSNs and it is a strong candidate for Smart Grid and Smart Home energy control and management applications. The advantage of ZigBee protocol is his operating on batteries in general contrary other protocols such as WiFi. Due to the low duty cycle mechanism, ZigBee can work for several years without the need for battery replacement. ZigBee is low in power, cost and complexity, and it can support up to 64000 nodes (devices) [3-4].

The layout of this paper is as follows: Section 2 describes the ZigBee Protocol. Section 3 discusses the proposed scenarios. Section 4 describes the RSSI calculation. Section 5 discusses the simulation results and, finally, the conclusion and future work are presented.

\section{ZIGBEE PROTOCOL}

The ZigBee communication protocol is popularly adopted in wireless sensor networks (WSNs) [5,6], because it has low-power and low-cost characteristics [7], and therefore it is very suitable for the development and use of Smart Home. The physical layer of ZigBee protocol supports three frequency bands; a $2450 \mathrm{MHz}$ band (with 16 channels), a $915 \mathrm{MHz}$ band (with 10 channels) and 868 $\mathrm{MHz}$ band (1 channel).

\section{TABLE I ZIGBEE FREQUENCY BANDS AND DATA RATES}

\begin{tabular}{|c|c|c|c|}
\hline Band & $\mathbf{8 6 8} \mathbf{~ M H z}$ & $\mathbf{9 1 5} \mathbf{~ M H z}$ & $\mathbf{2 4 5 0 M H z}$ \\
\hline Region & EU. Japan & USA & Worldwide \\
\hline Channels & 1 & 10 & 16 \\
\hline Data rate & $20 \mathrm{kbps}$ & $40 \mathrm{kbps}$ & $250 \mathrm{kbps}$ \\
\hline
\end{tabular}

\section{III.PROPOSED SCENARIOS}

Fig.1 below shows the tow scenarios of ZigBee communication inside the micro grid and the two-ways data flow between the micro grid and the Home Management and Control (HMC) system.

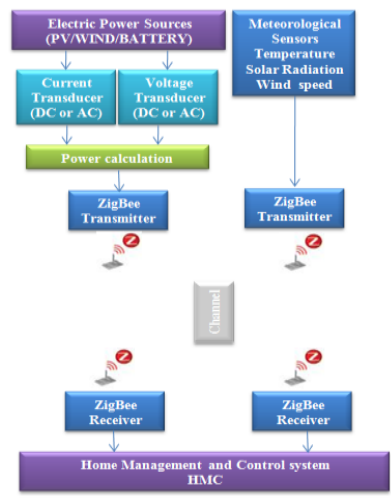

(a)

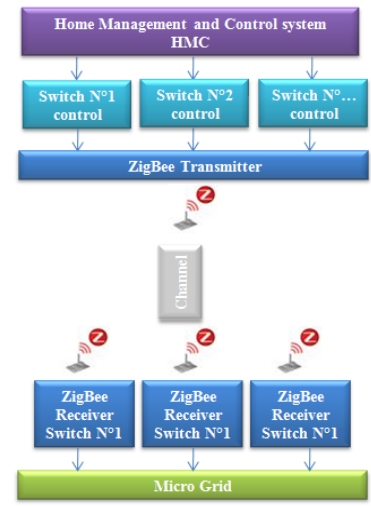

(b)
Fig.1 ZigBee communication process: (a) Micro Grid to HMC. (b) HMC to Micro Grid.

Fig. 2 illustrates the global scenario of WSN implanted in Opnet Modeler in order to simulate it. 
INTERNATIONAL JOURNAL OF INNOVATIVE RESEARCH IN ELECTRICAL, ELECTRONICS, INSTRUMENTATION AND CONTROL ENGINEERING Vol. 4, Issue 3, March 2016

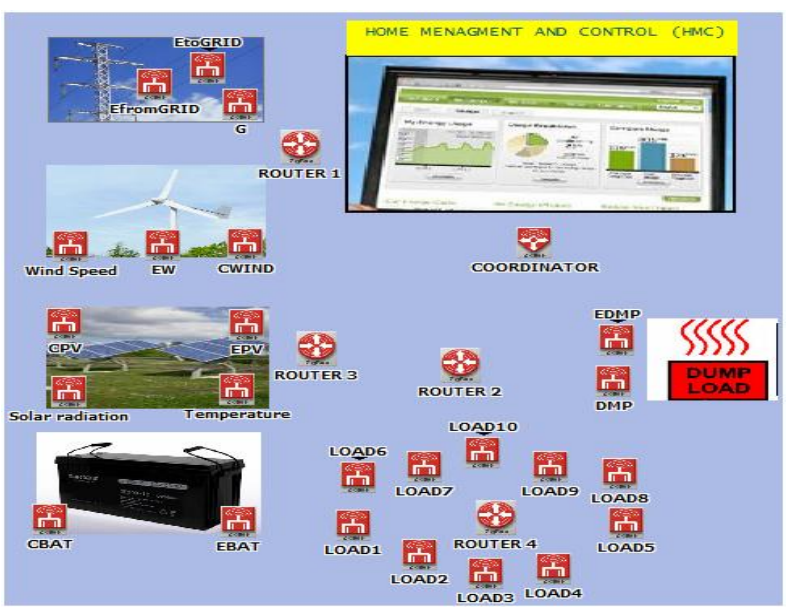

Fig. 2 Global scenario implanted in Opnet Modeler

\section{IV.RSSI CALCULATION}

The Received Signal Strength Indicator (RSSI) indicates the signal power at the receiving end, is used to estimate the distance between the WSN nodes. It is a parameter to identify the incoming radio signal. The free space transmission equation $[9,10,11]$ is given in Equation (1).

$$
\mathrm{P}_{\mathrm{RX}}=\mathrm{P}_{\mathrm{TX}} \cdot \mathrm{G}_{\mathrm{TX}} \cdot \mathrm{G}_{\mathrm{RX}} \cdot\left(\frac{\lambda}{4 \pi d}\right)^{2}
$$

Where;

$\mathrm{P}_{\mathrm{RX}}$ : Transmission power of sender

$\mathrm{P}_{\mathrm{TX}}$ : Remaining power of wave at receiver

$\mathrm{G}_{\mathrm{TX}}$ : Gain of transmitter

$\mathrm{G}_{\mathrm{RX}}$ : Gain of receiver

$\lambda:$ Wave length

$\mathrm{d}$ : Distance between sender and receiver

The Received Signal Strength (RSS) is converted to a Received Signal Strength Indicator (RSSI) in embedded devices, which is defined as ratio of the received power to the reference power $P_{\text {ref }}$ [12]. The typically reference power represents an absolute value of $\mathrm{P}_{\text {ref }}=1 \mathrm{~mW}$ [12]. The RSSI formula is shown as in Equation (2) as below;

RSSI $=10 . \log \left(\frac{P_{R X}}{\mathrm{P}_{\mathrm{r} e f}}\right)(\mathrm{dBm})$

\section{SIMULATION RESULTS AND DISCUSSION}

A. ZigBee simulation with OPNET Modeler OPNET Modeler is used to evaluate and compare the performance of different network configurations for our smart micro grid. The network performance is evaluated in terms of the following metrics:

- Latency, also known as End-to-End (ETE) delay: represents time (in seconds) for data to be delivered from source to destination.

- The network throughput: is the average rate of successful message delivery over a communication channel.

Three scenarios are simulated: star, tree and mesh topology. Table II describes the simulation parameters of all scenarios.

\section{TABLE III SIMULATION PARAMETERS}

\begin{tabular}{|l|c|}
\hline PARAMETER & VALUE \\
\hline Number of sensor nodes in PV system & 4 \\
\hline Number of sensor nodes in WIND system & 3 \\
\hline Number of sensor nodes in battery system & 2 \\
\hline Number of sensor nodes in loads (5 loads) & 5 \\
\hline Number of sensor nodes in dump load system & 2 \\
\hline Number of sensor nodes in grid system & 3 \\
\hline Data rate & $250 \mathrm{Kbps}$ \\
\hline Loads & 20 \\
\hline Simulation time & 1 hour \\
\hline
\end{tabular}

B. Simulation results with OPNET Modeler

Fig. 3 shows the MAC delay result of the three topologies. The star and the mesh topologies have near End-to-End delay in this simulation. The star topology has an End-toEnd delay. In this simulation, the tree topology has the highest global throughput (bits/second). The mesh topology has the second highest global throughput. Finally, the star topology has the lowest global throughput Fig 4.

Different scenarios are configured, simulated and compared in view of total end-to-end delay in Fig. 5 . The loads located in an area of $100 \mathrm{~m} \times 100 \mathrm{~m}$. The number of loads is considered 5, 10 and 20 loads. Fig. 6 shows that he throughput achieves in mesh topology

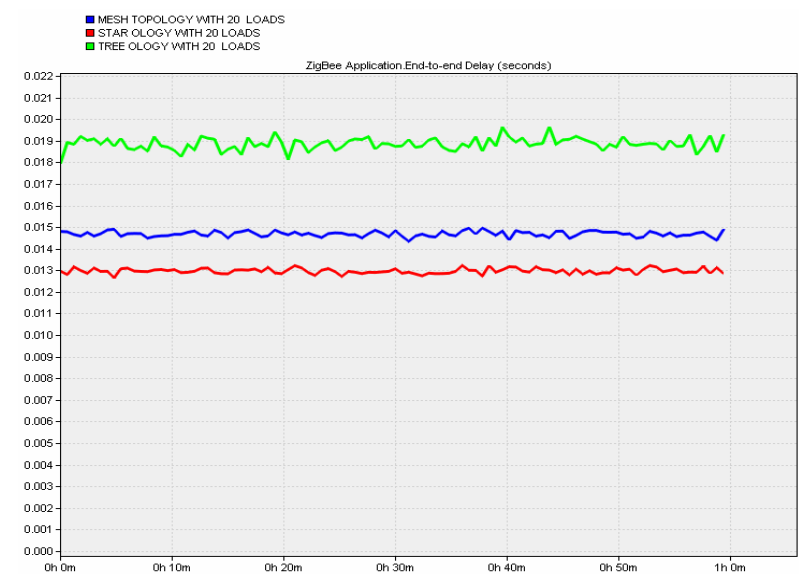

Fig. 3 Global scenario simulated with star, tree and mesh topology (20 loads).

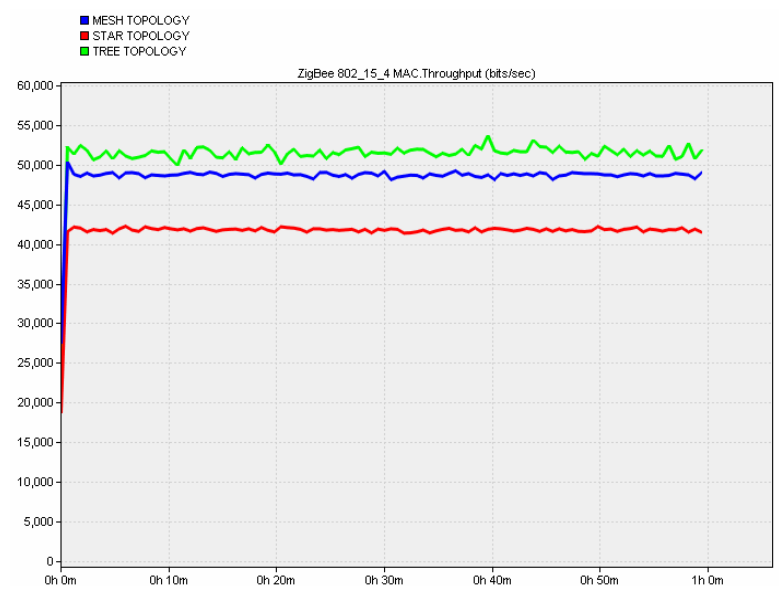

Fig. 4 Throughput for star, tree and mesh topology 


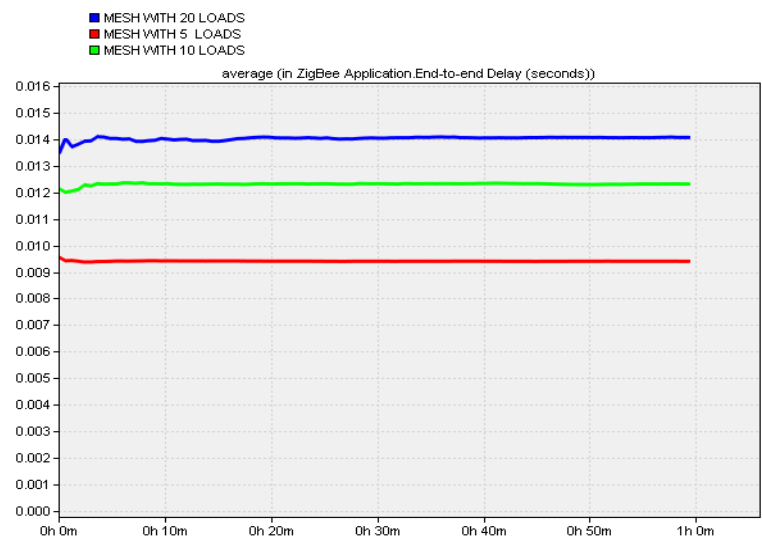

Fig. 5 Average End to End delay in mesh topology for 5 10 and 20 loads

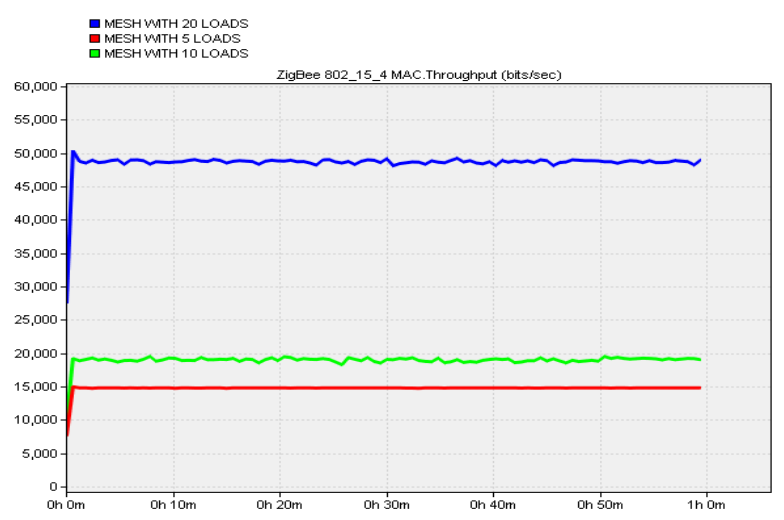

Fig. 6 Throughput in mesh topology for 5, 10 and 20 loads

C. Discussion results of Opnet simulation

The results show that tree topology outperforms all other topologies. Simulation results have shown that the tree topology has very effective throughput but is very slow in End-to-End Delay. This leads to more power consumption for the protocol and therefore the battery life is lower compared to star or mesh topology. For the smart home applications, the mesh topology will be better thanks to flexibility in network, adequate throughput and acceptable End-to-End delay. The impact number of load controller's communication performance shows that End-to-End Delay increases if the number of loads increases. It could be observed the End to End delay about $0.0140 \mathrm{~s}$ with 10 loads, $0.0122 \mathrm{~s}$ with 10 loads and $0.0094 \mathrm{~s}$ with 5 loads.

\section{Measuring of RSSI}

The measurements consist to detect the average value of RSSI obtained from the reception of 100 messages. Each measurement is an average of 10 repetitions of the same experiment to counteract the signal fluctuations

TABLE IIIII SIGNAL LOSS IN FREE SPACE

\begin{tabular}{|c|c|}
\hline $\begin{array}{c}\text { Distance between } \\
\text { tow nodes }\end{array}$ & $\begin{array}{c}\text { Average Attenuation } \\
\text { RSSI (dB) }\end{array}$ \\
\hline $50 \mathrm{~cm}$ & 0.00 \\
\hline $2 \mathrm{~m}$ & 11.32 \\
\hline $3 \mathrm{~m}$ & 14.01 \\
\hline $6 \mathrm{~m}$ & 21.07 \\
\hline $9 \mathrm{~m}$ & 25.15 \\
\hline $11 \mathrm{~m}$ & 29.89 \\
\hline
\end{tabular}

caused by inside discoloration. The experiments have been performed in an indoor environment with both Line-OfSight (LOS) and Non Line-Of-Sight (NLOS) conditions. The results are presented in TABLE III and TABLE IV.

TABLE IVV SIGNAL LOSS WITH OBSTACLES

\begin{tabular}{|l|c|}
\hline \multicolumn{1}{|c|}{ Obstacles } & $\begin{array}{c}\text { Average Attenuation } \\
\text { RSSI (dB) }\end{array}$ \\
\hline Window (metal stores open) & 1.01 \\
\hline Window (metal Stores closed) & 3.82 \\
\hline Wall with door open & 0.31 \\
\hline Wall with door closed & 1.28 \\
\hline Wall with Brick & 1.52 \\
\hline Between two floors & 13.87 \\
\hline
\end{tabular}

The obtained measurements show that other than the distance that separates two nodes, there are few factors that degrade and have a negative impact on the RSSI values in ZigBee communication network as reflections on metal objects, diffraction at edges, floors existing, etc...

\section{VI.CONCLUSION AND FUTURE WORKS}

In this work, simulation results with Opnet Modeler and RSSI measurements concluded that the ZigBee technology may be very promising in Smart Homes, Smart Micro Grids and Smart Grids applications such as the energy control and management. Future research will be based on the possibility of coexistence between ZigBee protocol and other protocols operate at the same frequency bands such as Wifi and Bluetooth.

\section{REFERENCES}

[1] N. Batista; et al. (2013). Photovoltaic and wind energy systems monitoring and building/home energy management using ZigBee devices within a smart grid. Energy, Elsevier, 49, 306-315.

[2] J.Higuera; E. Kartsakli; J.L. Valenzuela; L. Alonso; A. Laya, R. Martinez; A. Aguilar. (2012) Experimental Study of Bluetooth, ZigBee and IEEE 802.15.4 Technologies on Board High-Speed Trains. Vehicular Technology Conference (VTC Spring), IEEE, 75th, 1- 5

[3] N. Batista; et al. "Photovoltaic and wind energy systems monitoring and building/home energy management using ZigBee devices within a smart grid." Energy, Elsevier, doi:10.1016/j.energy. 2012.11.002, vol. 49, pp. 306-315, 2013.

[4] J.Higuera; E. Kartsakli; J.L. Valenzuela; L. Alonso; A. Laya, R. Martinez; A. Aguilar. "Experimental Study of Bluetooth, ZigBee and IEEE 802.15.4 Technologies on Board High-Speed Trains." IEEE Vehicular Technology Conference (VTC Spring), IEEE, DOI:10.1109/VETECS.2012.6239971, 75, pp. 1- 5, 2012.

[5] Zhenyu Zou and al. "Smart Home System Based on IPV6 and ZIGBEE Technology", Advanced in Control Engineering and Information Science, Published by Elsevier Ltd, pp. 1529-1533, 2011

[6] Chia-Wen Lu, "Interconnecting ZigBee and 6LoWPAN wireless sensor networks for smart grid applications", Fifth IEEE International Conference on Sensing Technology (ICST), 2011, pp. 267 - 272.

[7] Dongmei Yan, "ZigBee-based Smart Home system design" Advanced Computer Theory and Engineering (ICACTE), 3rd IEEE International Conference, Aug. 2010, pp V2-650 - V2-653.

[8] Xbee®/XBee-Pro® ZB OEM RF Modules Manual, ver. 4/14/2008: http://ftp1.digi.com/support/documentation/90000976 a.pdf

[9] Theodore S. Rappaport, "Wireless Communications: Principles and Practice”. New Jersey: Prentice-Hall Inc., 1996.

[10] Maissa Ben Jam^aa, Anis Koub^aa, Yasir Kayani, "EasyLoc: RSSbased Localization Made Easy", The International Workshop on Cooperative Robots and Sensor Networks (RoboSense), 2012, pp. 1127 - 1133

[11] Potortì F, Corucci A, Nepa P, Furfari F, Barsocchi P, Buffi A, "Accuracy limits of in-room localisation using RSSI", proceedings of the Antennas and Propagation Society International Symposium; 2009

[12] Theodore S. Rappaport, "Wireless Communications: Principles and Practice”. New Jersey: Prentice-Hall Inc., 1996. 